\title{
What is the Complexity of Related Elliptic, Parabolic, and Hyperbolic Problems?
}

\author{
By Arthur G. Werschulz*
}

\begin{abstract}
Traub and Woźniakowski have dealt with the complexity of some simple partial differential equations. They chose three model problems and showed that the parabolic problem considered had significantly lower complexity than the elliptic problem, which in turn had significantly lower complexity than the hyperbolic problem considered. They asked whether this is true in general. We show that this is not the case by proving that if $L$ is a reasonably well-behaved elliptic operator, then the steadystate heat equation $L u=f$, the heat equation $\partial_{t} u+L u=f$, and the wave equation $\partial_{t t} u+L u=f$ all have roughly the same worst-case complexity for $f$ in the unit ball of a certain Sobolev space of smoothness $r$.
\end{abstract}

1. Introduction. This paper deals with the complexity of "related" elliptic, parabolic, and hyperbolic partial differential equations. (In this Introduction we use terms such as complexity, minimal error, etc., without definition; they are defined rigorously later.)

Traub and Woźniakowski [9] have dealt with the complexity of three partial differential equations. The first problem was the heat equation on a thin rod of length $\pi$, with zero boundary data; the initial data was odd of period $2 \pi$, having an $r$ th derivative whose $L_{2}$-norm was bounded by unity. They found that if the solution was considered at a fixed time $t_{0}$, then the $n$th minimal error in the $L_{2}$ norm was $e^{-(n+1)^{2} t_{0}} /(n+1)^{r}$, and the complexity of finding an $\varepsilon$-approximation was $\Theta\left(\left(\ln \varepsilon^{-1}\right)^{1 / 2}\right)$ as $\varepsilon \rightarrow 0$. (The $\Theta$-notation used here is that of [7]; it may be thought of as a two-sided $O$-notation.)

The second problem was Laplace's equation on a square in the $(x, y)$-plane, the length of whose sides was $\pi$. Zero boundary data was prescribed on the west, south, and east sides of the square; the boundary data on the north side satisfied the same conditions as the initial data in the heat equation above. If the solution was considered along a line $y=y_{0}$ (where $0<y_{0}<\pi$ ), then they found the $n$th minimal $L_{2}$-error to behave asymptotically as $e^{-(n+1)\left(\pi-y_{0}\right)} /(n+1)^{r}$, and the complexity of finding an $\varepsilon$-approximation to be $\Theta\left(\ln \varepsilon^{-1}\right)$ as $\varepsilon \rightarrow 0$.

Received October 25, 1983; revised January 13, 1986.

1980 Mathematics Subject Classification. Primary 65M15, 65N15, 68C25; Secondary 35J40, $35 \mathrm{~K} 25,35 \mathrm{~L} 25,65 \mathrm{M} 60,65 \mathrm{~N} 30$.

Key words and phrases. Optimal solution of partial differential equations, elliptic problems, parabolic problems, hyperbolic problems, computational complexity, information-based complexity, optimal algorithms.

*This research was supported in part by the National Science Foundation under Grants MCS8203271 and MCS-8303111.

This paper was prepared by the author using $A_{M} S-T_{E X}$ and transmitted electronically to the AMS for final typesetting. 
The third problem was the first-order hyperbolic equation

$$
\frac{\partial u}{\partial t}=\frac{\partial u}{\partial x}
$$

The initial data had period $2 \pi$ and mean value zero, with an $r$ th derivative whose $L_{2}$-norm was bounded by unity. They considered the solution $u\left(\cdot, t_{0}\right)$ on the interval $[0, \pi]$, for a fixed $t_{0}>0$. They found the $n$th minimal $L_{2}$-error to be $(\lfloor n / 2\rfloor+1)^{-r}$, and the complexity of finding an $\varepsilon$-approximation to be $\Theta\left(\varepsilon^{-1 / r}\right)$ as $\varepsilon \rightarrow 0$.

Hence, they found examples of parabolic, elliptic, and hyperbolic problems for which the parabolic problem had significantly smaller complexity than the elliptic problem, which, in turn, had significantly smaller complexity than the hyperbolic problem. They asked $[9$, p. 149] whether this was true in general, or whether this depended on these specially chosen examples.

In this paper, we show that this phenomenon is not true in general.

We first note that the behavior noted in [9] is norm-dependent. Traub and Woźniakowski showed that the $n$th minimal error for all three problems became $\Theta\left(n^{-r}\right)$ as $n \rightarrow \infty$, when the error was measured in the $L_{\infty}\left(L_{2}\right)$ sense, and the complexity of finding an $\varepsilon$-approximation became $\Theta\left(\varepsilon^{-1 / r}\right)$ as $\varepsilon \rightarrow 0$. However, the $L_{\infty}\left(L_{2}\right)$-norm is not a natural way to measure the error for the elliptic problem, since it gives more importance to the $y$-direction than it does to the $x$-direction.

Another difficulty is that the class of problem elements either played a different role or changed when going from one problem to another. The problem elements were initial data for the parabolic and hyperbolic problems, while they were boundary data for the elliptic problem. Moreover, they were odd functions in the parabolic and hyperbolic problems, whereas they were functions with zero mean in the elliptic problem. Hence, the notion of "a class of problem elements with smoothness $r$ " changed from problem to problem.

In this paper, we are interested in the inherent complexity of related elliptic, parabolic, and hyperbolic problems. That is, we let $L$ be a reasonably well-behaved elliptic operator of order $2 m$. For a smooth region $\Omega \subset \mathbf{R}^{N}$, we will let $\dot{H}^{r}(\Omega)$ denote a closed subspace of $H^{r}(\Omega)$-functions satisfying certain boundary conditions (which will be specified later). We then consider the elliptic problem $L u=f$, the parabolic problem $\partial_{t} u+L u=f$, and the hyperbolic problem $\partial_{t t} u+L u=f$ for all $f$ in the unit ball of $\dot{H}^{r}(\Omega)$. Note that the same operator $L$ appears in all three problems. Hence, these problems are related in the same way that the classical Poisson, heat, and wave equations are related. Moreover, the problem element $f$ plays the same role in all three problems. Errors will be measured in the $L_{2}$-norm for the elliptic problem, and either the $L_{2}$-norm at a fixed time $t_{0}$ or the $L_{\infty}\left(L_{2}\right)$-norm for the time-dependent problems.

Our main result is that for the elliptic and parabolic problems and for the hyperbolic problem solved over an interval in time, the $n$th minimal error is $\Theta\left(n^{-(r+2 m) / N}\right)$ as $n \rightarrow \infty$, and the complexity of finding an $\varepsilon$-approximation is $\Theta\left(\varepsilon^{-N /(r+2 m)}\right)$ as $\varepsilon \rightarrow 0$. For the hyperbolic problem solved at a particular time $t_{0}$, the " $\Theta$ " becomes an " $O$ "; moreover, this is (roughly speaking) the strongest statement possible, since a fortuitous value of $t_{0}$ could make the $n$th minimal error zero for all $n$.

It is important to point out that these results are mainly of theoretical interest. There are two reasons for this. 
(i) We assume that the problem element $f$ belongs to $\dot{H}^{r}(\Omega)$, and thus satisfies some boundary conditions. It is more usual to assume only that $f \in H^{r}(\Omega)$, i.e., that $f$ satisfies no boundary conditions (see, e.g., [4] and [8]).

(ii) We are mainly interested in the inherent (or intrinsic) complexity of these problems, which allows us to consider algorithms that may not be implementable in practice. For example, we assume a model of computation permitting infiniteprecision arithmetic and for which exact information is available; if either of these assumptions is weakened, the complexity of these problems might change. In addition, we consider algorithms using information that might not be readily available, such as the eigenvalues and eigenfunctions of $L$.

However, knowing the inherent complexity does provide a benchmark; it tells what price the user is paying for using nonoptimal information or nonoptimal algorithms. In addition, we are able to show that there are finite element methods that are nearly optimal, so that if the usual "finite element information" [10] is available, these results become useful in practice.

We now outline the contents of this paper. In Section 2, we define the problems to be studied. In Section 3, we recall some terminology and results from [9] about optimal algorithms. In Section 4, we compute $n$th minimal errors for these related problems and show that they are roughly the same. In Section 5, these results are used to show that the complexity of finding $\varepsilon$-approximations is roughly the same for all three problems. Finally, we summarize our results and pose some open questions in Section 6.

2. Related Elliptic, Parabolic, and Hyperbolic Problems. In this section, we define the problems to be studied. We use the standard terminology and notations found in [8] for multi-indices, Sobolev spaces, etc.

Given a positive integer $N$, let $\Omega \subset \mathrm{R}^{N}$ be a bounded region with $C^{\infty}$ boundary. Consider the formally selfadjoint, uniformly strongly $2 m$ th-order elliptic operator $L$ of the form

$$
(L v)(x)=\sum_{|\alpha|,|\beta| \leq m}(-1)^{\alpha} D^{\alpha}\left(a_{\alpha \beta}(x) D^{\beta} v(x)\right),
$$

with real-valued functions $a_{\alpha \beta} \in C^{\infty}(\bar{\Omega})$ such that $a_{\alpha \beta}=a_{\beta \alpha}$. We additionally assume that

(2.1) $L v=0$ in $\Omega$ and $\partial_{\nu}^{j} v=0$ on $\partial \Omega$ (for $\left.0 \leq j \leq m-1\right) \Rightarrow v=0$ in $\Omega$,

where $\partial_{\nu}$ denotes the outer normal derivative on $\partial \Omega$.

To define the space of problem elements, we recall the following result. Let $\beta_{1}$, $\beta_{2}, \ldots$ denote the eigenvalues (arranged in nondecreasing order) and let $z_{1}, z_{2}, \ldots$ denote the corresponding $L_{2}(\Omega)$-orthonormal eigenfunctions of the problem

$$
\begin{aligned}
L z_{n} & =\beta_{n} z_{n} & & \text { in } \Omega, \\
\partial_{\nu}^{j} z_{n} & =0 & & \text { on } \partial \Omega(0 \leq j \leq m-1) .
\end{aligned}
$$

Then [1, Theorem 4.6] shows that there is a positive constant $c$, which is independent of $n$, such that

$$
\beta_{n} \sim c n^{2 m / N} \text { as } n \rightarrow \infty
$$

and so $\lim _{n \rightarrow \infty} \beta_{n}=\infty$.

Let $r \geq 0$. Following the approach of [2], we now define the space $\dot{H}^{r}(\Omega)$ of problem elements to be

$$
\dot{H}^{r}(\Omega)=\left\{f \in L_{2}(\Omega):\|f\|_{r}<\infty\right\}
$$


where the norm $\|\cdot\|_{r}$ is defined by

$$
\|f\|_{r}=\sqrt{\sum_{n=1}^{\infty} \beta_{n}^{r / m}\left(f, z_{n}\right)^{2}} \equiv\left\|L^{r / 2 m} f\right\|_{L_{2}(\Omega)} .
$$

(Here and in the rest of this paper, $(\cdot, \cdot)$ denotes the $L_{2}(\Omega)$-inner product.) Using elliptic regularity theory (see [8, Theorem 5.6]) and Hilbert space interpolation theory (see [3, Chapter III] and [6]), it is easy to see that $\dot{H}^{r}(\Omega)$ is a closed subspace of the Sobolev space $H^{r}(\Omega)$, and that the norm $\|\cdot\|_{r}$ is equivalent to the usual Sobolev norm $\|\cdot\|_{H^{r}(\Omega)}$ on $\dot{H}^{r}(\Omega)$.

We are interested in the complexity of the following elliptic, parabolic, and hyperbolic problems:

Problem (E). Given $f \in \dot{H}^{r}(\Omega)$, find $u: \bar{\Omega} \rightarrow \mathbf{R}$ such that

$$
\begin{aligned}
L u=f & \text { in } \Omega, \\
\partial_{\nu}^{j} u=0 & \text { on } \partial \Omega(0 \leq j \leq m-1) .
\end{aligned}
$$

We define

$$
S_{E} f:=u \text {. }
$$

Note that (2.1) implies that $S_{E} f$ is defined for all $f \in \dot{H}^{r}(\Omega)$.

In the next two problems, we fix a value of $T>0$.

Problem (P). Given $f \in \dot{H}^{r}(\Omega)$, find $u: \bar{\Omega} \times[0, T] \rightarrow \mathbf{R}$ such that

$$
\begin{aligned}
\partial_{t} u+L u=f & \text { in } \Omega \times(0, T), \\
\partial_{\nu}^{j} u=0 & \text { on } \partial \Omega \times[0, T](0 \leq j \leq m-1), \\
u(\cdot, 0)=0 & \text { on } \Omega .
\end{aligned}
$$

If we wish the solution for all $t \in[0, T]$, we will consider

$$
S_{P} f:=u \text {. }
$$

If we are interested in the solution at a particular $t_{0} \in[0, T]$, we will consider

$$
S_{P, t_{0}} f:=u\left(\cdot, t_{0}\right)
$$

Problem $(\mathrm{H})$. Given $f \in \dot{H}^{r}(\Omega)$, find $u: \bar{\Omega} \times[0, T] \rightarrow \mathrm{R}$ such that

$$
\begin{aligned}
& \partial_{t t} u+L u=f \text { in } \Omega \times(0, T), \\
& \partial^{j} u=0 \text { on } \partial \Omega \times[0, T](0 \leq j \leq m-1), \\
& u(\cdot, 0)=\partial_{t} u(\cdot, 0)=0 \quad \text { on } \Omega .
\end{aligned}
$$

If we wish the solution for all $t \in[0, T]$, we will consider

$$
S_{H} f:=u \text {. }
$$

If we are interested in the solution at a particular $t_{0} \in[0, T]$, we will consider

$$
S_{H, t_{0}} f:=u\left(\cdot, t_{0}\right) \text {. }
$$

The problems (E), (P), and $(\mathrm{H})$ are said to be related because they all involve the same elliptic operator $L$. Note that if $-L$ is the $N$-dimensional Laplacian, then $(\mathrm{E})$ becomes Poisson's equation, while $(\mathrm{P})$ and $(\mathrm{H})$ become the heat and wave equations (respectively) with a forcing term. 
3. Optimal-Error Algorithms and Optimal Information. In this section, we introduce some terminology and results from [9] about optimal-error algorithms using given information, as well as the selection of optimal information. (See [9] for further discussion of these concepts.)

Let $S: \dot{H}^{r}(\Omega) \rightarrow G$ be a linear transformation. We call $S$ a solution operator. When $S$ is one of $S_{E}, S_{P, t_{0}}$, or $S_{H, t_{0}}$, we will choose $G=L_{2}(\Omega)$; when $S$ is either $S_{P}$ or $S_{H}$, we will let $G=L_{\infty}\left([0, T], L_{2}(\Omega)\right)$ under the norm

$$
\|u\|_{L_{\infty}\left(L_{2}\right)}=\underset{0 \leq t \leq T}{\operatorname{ess} \sup }\|u(\cdot, t)\|_{L_{2}(\Omega)} .
$$

We will often refer to the problem for which $S$ is the solution operator as the problem $S$.

Let $\varepsilon>0$. Our main goal is to find an $\varepsilon$-approximation to the problem $S$. In other words, let $F$ denote the unit ball of $\dot{H}^{r}(\Omega)$. Then for $f \in F$, we wish to compute $x(f) \in G$ such that

$$
\|S f-x(f)\|_{G}<\varepsilon .
$$

In what follows, we will sometimes refer to $F$ as the set of problem elements.

To find an $\varepsilon$-approximation, we must know something about the problem elements. We assume that for any $f \in F$, we know only a finite amount of "information" $N f$ about $f$, consisting of the values of $n$ linear functionals at $f$, i.e.,

$$
N f=\left[\begin{array}{c}
\lambda_{1}(f) \\
\vdots \\
\lambda_{n}(f)
\end{array}\right] \quad \forall f \in \dot{H}^{r}(\Omega) .
$$

Hence, information $N$ of cardinality at most $n$ is a linear operator $N: \dot{H}^{r}(\Omega) \rightarrow \mathrm{R}^{n}$.

We must now use this information in an algorithm, i.e., a mapping $\varphi: \mathbf{R}^{n} \rightarrow G$. The quality of an algorithm $\varphi$ using information $N$ to find an approximate solution of the problem $S$ is measured by its (worst-case) error

$$
e(\varphi, N, S)=\sup _{f \in F}\|S f-\varphi(N f)\|_{G}
$$

Clearly, an algorithm $\varphi$ using information $N$ gives an $\varepsilon$-approximation to the problem $S$ if and only if $e(\varphi, N, S)<\varepsilon$.

Of course, we wish to use the information $N$ as efficiently as possible. Let the radius of information

$$
r(N, S)=\inf _{\varphi} e(\varphi, N, S)
$$

be the minimal error among all algorithms using $N$ for the problem $S$. We seek an optimal-error algorithm $\varphi_{N}$ using $N$, i.e., an algorithm $\varphi_{N}$ using $N$ such that

$$
e\left(\varphi_{N}, N, S\right)=r(N, S)
$$

(See [9] for an explanation of the term "radius of information," as well as for further discussion of optimal-error algorithms.)

Finally, we are interested in selecting the best information of given cardinality for the problem $S$. Let

$$
r(n, S)=\inf \{r(N, S): N \text { is information of cardinality at most } n\}
$$


denote the $n$th minimal radius of information for the problem $S$, so that there is no information of cardinality at most $n$ whose radius is less than $r(n, S)$. An information operator $N_{n}$ of cardinality at most $n$ is said to be $n$th optimal information for the problem $S$ if

$$
r\left(N_{n}, S\right)=r(n, S)
$$

Of course, if $\varphi_{n}$ is an optimal-error algorithm using $n$th optimal information $N_{n}$, then

$$
e\left(\varphi_{n}, N_{n}\right) \leq e(\varphi, N)
$$

for any algorithm $\varphi$ using any information $N$ of cardinality at most $n$. Such an algorithm $\varphi_{n}$ is called an $n$th minimal-error algorithm for the problem $S$.

Remark 3.1. Note that we only consider linear information that is nonadaptive, i.e., the linear functional $\lambda_{i}$ does not depend on the previously computed information. One might also consider adaptive information, in which the linear functional $\lambda_{i}$ depends on the values of $\lambda_{1}, \ldots, \lambda_{i-1}$. For the problems considered in this paper, adaptive and nonadaptive linear information are equally powerful (see [9, Chapter 2]). In addition, one might consider nonlinear information, in which $\lambda_{1}, \ldots, \lambda_{n}$ are nonlinear functionals. It is known that arbitrary nonlinear information is too powerful (see [9, Chapter 7]). However, continuous nonlinear information is no more powerful than linear information (see [5]).

To see an example of $n$th optimal information and an $n$th minimal-error algorithm, suppose that the solution operator $S: \dot{H}^{r}(\Omega) \rightarrow G$ has the form

$$
S f=\sum_{i=1}^{\infty} \sigma_{i}\left(f, z_{i}\right) z_{i} \quad \forall f \in \dot{H}^{r}(\Omega),
$$

where $\left\{z_{i}\right\}_{i=1}^{\infty}$ is an orthonormal basis for $\dot{H}^{r}(\Omega)$. Let

$$
\lambda_{i}=\frac{\sigma_{i}}{\beta_{i}^{r / 2 m}} \quad(i=1,2, \ldots),
$$

and suppose that $\lambda_{i_{j}}$ is the $j$ th largest value of $\left\{\lambda_{i}\right\}_{i=1}^{\infty}$, i.e., $\lambda_{i_{1}} \geq \lambda_{i_{2}} \geq \cdots \geq 0$. Then [6, Theorem 6.6.1] immediately gives

LEMMA 3.1. (i) The nth minimal radius for the problem $S$ is

$$
r(n, S)=\lambda_{i_{n+1}} .
$$

(ii) The information $N_{n}$ given by

$$
N_{n} f=\left[\begin{array}{c}
\left(f, z_{i_{1}}\right) \\
\vdots \\
\left(f, z_{i_{n}}\right)
\end{array}\right] \quad \forall f \in \dot{H}^{r}(\Omega)
$$

is nth optimal information for the problem $S$.

(iii) The algorithm $\varphi_{n}$ given by

$$
\varphi_{n}\left(N_{n} f\right)=\sum_{j=1}^{n}\left(f, z_{i_{j}}\right) z_{i_{j}} \quad \forall f \in \dot{H}^{r}(\Omega)
$$

is an nth minimal-error algorithm for the problem $S$.

This result will be useful in determining optimal information and minimal-error algorithms for the problems (E), $(\mathrm{P})$, and $(\mathrm{H})$. 
4. Minimal-Error Algorithms for Related Problems. In this section, we use Lemma 3.1 to show that the $n$th minimal errors for the related problems are all roughly the same. Moreover, the same type of information is $n$th optimal information for all three problems. We also give $n$th minimal-error algorithms for these problems.

4.1. Elliptic Problem $S_{E}$. Since $\left\{z_{j}\right\}_{j=1}^{\infty}$ is an orthonormal basis of eigenfunctions for $L$ corresponding to the eigenvalues $\left\{\beta_{j}\right\}_{j=1}^{\infty}$ with $0<\beta_{1} \leq \beta_{2} \leq \cdots$, we see that

$$
S_{E} f=\sum_{j=1}^{\infty} \frac{1}{\beta_{j}}\left(f, z_{j}\right) z_{j} \quad \forall f \in \dot{H}^{r}(\Omega) .
$$

(Recall that $(\cdot, \cdot)$ denotes the $L_{2}(\Omega)$-inner product.) Using $(2.2)$ and Lemma 3.1, we have

THEOREM 4.1. (i) The $n$th minimal radius of information for the elliptic problem $S_{E}$ is

$$
r\left(n, S_{E}\right)=\beta_{n+1}^{-(r+2 m) /(2 m)}
$$

so that

$$
r\left(n, S_{E}\right)=\Theta\left(n^{-(r+2 m) / N}\right) \quad \text { as } n \rightarrow \infty .
$$

(ii) The information $N_{n}$, given by

$$
N_{n} f=\left[\begin{array}{c}
\left(f, z_{1}\right) \\
\vdots \\
\left(f, z_{n}\right)
\end{array}\right] \quad \forall f \in \dot{H}^{r}(\Omega),
$$

is nth optimal information for the elliptic problem $S_{E}$.

(iii) The algorithm $\varphi_{n}$, given by

$$
\varphi_{n}\left(N_{n} f\right)=\sum_{j=1}^{n} \frac{1}{\beta_{j}}\left(f, z_{j}\right) z_{j} \quad \forall f \in \dot{H}^{r}(\Omega),
$$

is an nth minimal-error algorithm for the elliptic problem $S_{E}$.

Of course, it will usually be difficult to determine the eigenfunctions and eigenvalues of operators $L$ arising in practice. Since our goal is to show that related problems have the same intrinsic complexity, this difficulty does not interfere with the aim of this paper. However, consider the finite element method using piecewise polynomial $n$-dimensional subspaces of $\dot{H}^{r}(\Omega)$, the degree of the polynomials being $\lceil r\rceil+2 m-1$. If a quasi-uniform family of triangulations is used, then the results of [4], [8], [10] imply that this finite element method has error $\Theta\left(n^{-(r+2 m) / N}\right)$ as $n \rightarrow \infty$. Hence, this finite element method is (to within a constant that is independent of $n$ ) an $n$th minimal-error algorithm for the elliptic problem $S_{E}$.

4.2. Parabolic Problems $S_{P, t_{0}}$ and $S_{P}$. Using separation of variables, we see that

$$
S_{P, t_{0}} f=\sum_{j=1}^{\infty} \frac{1-e^{-\beta_{j} t_{0}}}{\beta_{j}}\left(f, z_{j}\right) z_{j} \quad \forall f \in \dot{H}^{r}(\Omega) .
$$


Using (2.2), Lemma 3.1, and the techniques of [9, pp. 59-60], we have

THEOREM 4.2. (i) The nth minimal radius of information for the parabolic problem $S_{P, t_{0}}$ at time $t_{0}$ is

$$
r\left(n, S_{P, t_{0}}\right)=\left(1-e^{-\beta_{n+1} t_{0}}\right) \beta_{n+1}^{-(r+2 m) /(2 m)},
$$

while the nth minimal radius of information for the parabolic problem $S_{P}$ over the interval $[0, T]$ is

$$
r\left(n, S_{P}\right)=\beta_{n+1}^{-(r+2 m) /(2 m)}
$$

Hence,

$$
r\left(n, S_{P, t_{0}}\right)=\Theta\left(n^{-(r+2 m) / N}\right) \quad \text { as } n \rightarrow \infty
$$

and

$$
r\left(n, S_{P}\right)=\Theta\left(n^{-(r+2 m) / N}\right) \quad \text { as } n \rightarrow \infty .
$$

(ii) The information $N_{n}$ given by (4.1) is nth optimal information for the parabolic problems $S_{P, t_{0}}$ and $S_{P}$.

(iii) Let

$$
\varphi_{n}\left(N_{n} f\right)(t)=\sum_{j=1}^{n} \frac{1-e^{-\beta_{j} t}}{\beta_{j}}\left(f, z_{j}\right) z_{j} \quad \forall(f, t) \in \dot{H}^{r}(\Omega) \times[0, T] .
$$

Then $\varphi_{n}(\cdot)\left(t_{0}\right)$ is an nth minimal-error algorithm for the parabolic problem $S_{P, t_{0}}$, and $\varphi_{n}$ is an nth minimal-error algorithm for the parabolic problem $S_{P}$.

Once again, the algorithm presented in this theorem will usually be difficult to implement in practice. However, the formula for $S_{P, t_{0}}$ may be used to derive shift theorems for $u$ and $\partial u / \partial t$. Following the results in [4, pp. $139 \mathrm{ff}$.], this means that the finite element method is optimal (to within a constant) for the parabolic problem. (Note that the techniques in [4] are only applied to the second-order problem. However, these techniques are related to those of Wheeler [11], who maintains that they extend to the general $2 m$ th-order problem.)

4.3. Hyperbolic Problems $S_{H, t_{0}}$ and $S_{H}$. Once again, using separation of variables, we find that

$$
S_{H, t_{0}} f=\sum_{j=1}^{\infty} \frac{1-\cos \left(\sqrt{\beta_{j}} t_{0}\right)}{\beta_{j}}\left(f, z_{j}\right) z_{j} \quad \forall f \in \dot{H}^{r}(\Omega) .
$$

We first consider the problem $S_{H, t_{0}}$. Let

$$
\rho_{i}=1-\cos \left(\sqrt{\beta_{i}} t_{0}\right)
$$

and let

$$
\lambda_{i}=\rho_{i} \beta_{i}^{-(r+2 m) /(2 m)} .
$$

Let $\lambda_{i_{j}}$ be the $j$ th largest value in the set $\left\{\lambda_{i}\right\}_{i=1}^{\infty}$, i.e., $\lambda_{i_{1}} \geq \lambda_{i_{2}} \geq \cdots>0$. Using (2.2) and Lemma 3.1, we have

THEOREM 4.3. (i) The nth minimal radius of information for the hyperbolic problem $S_{H, t_{0}}$ at time $t_{0}$ is

$$
r\left(n, S_{H, t_{0}}\right)=\lambda_{i_{n+1}}
$$

so that

$$
r\left(n, S_{H, t_{0}}\right)=O\left(n^{-(r+2 m) / N}\right) \quad \text { as } n \rightarrow \infty
$$


(ii) The information $N_{n}$ given by

$$
N_{n} f=\left[\begin{array}{c}
\left(f, z_{i_{1}}\right) \\
\vdots \\
\left(f, z_{i_{n}}\right)
\end{array}\right] \quad \forall f \in \dot{H}^{r}(\Omega)
$$

is $n$th optimal information for the hyperbolic problem $S_{H, t_{0}}$.

(iii) The algorithm $\varphi_{n}$ given by

$$
\varphi_{n}\left(N_{n} f\right)=\sum_{j=1}^{n} \frac{1-\cos \left(\sqrt{\beta_{i_{j}}} t_{0}\right)}{\beta_{i_{j}}}\left(f, z_{i_{j}}\right) z_{i_{j}} \quad \forall f \in \dot{H}^{r}(\Omega)
$$

is an nth minimal-error algorithm for the hyperbolic problem $S_{H, t_{0}}$.

The result for this problem is not as satisfying as those for the previous problems. There are two reasons why this is so. First, the $n$th optimal information and the $n$th minimal-error algorithm depend strongly on the ordering of the $\lambda_{j}$, which in turn depends strongly (and chaotically) on the value of $t_{0}$. Hence, a slight change in $t_{0}$ can greatly change the minimal-error algorithm for the problem $S_{H, t_{0}}$.

A second reason why this result is less satisfying is that we only have an upper bound of $O\left(n^{-(r+2 m) / N}\right)$ for the $n$th minimal radius for the problem $S_{H, t_{0}}$, whereas we had upper and lower bounds of $\Theta\left(n^{-(r+2 m) / N}\right)$ for the other problems. We would like to change this $O$-estimate to a $\theta$-estimate for the problem $S_{H, t_{0}}$. Unfortunately, this is not possible. The $O$-estimate is (roughly speaking) the strongest result that we can obtain, since a fortuitous value of $t_{0}$ can yield zero error for the zero algorithm $\varphi \equiv 0$, as in

Example 4.1. Let $N=1, \Omega=(0, \pi), m=1$, and $L v=-v^{\prime \prime}$. Then

$$
z_{n}(x)=\sqrt{\frac{2}{\pi}} \sin n x
$$

is the $n$th orthonormal eigenfunction of $L$ corresponding to the eigenvalue $\beta_{n}=n^{2}$. Hence

$$
S_{H, t_{0}} f=\sum_{j=1}^{\infty} \frac{1-\cos \left(j t_{0}\right)}{j^{2}}\left(f, z_{j}\right) z_{j}
$$

Suppose that $T \geq 2 \pi$. Then, setting $t_{0}=2 \pi$, we see that $\cos \left(j t_{0}\right)=1$ for any integer $j$, and so $S_{H, 2 \pi}$ is identically zero. Hence, the zero algorithm has zero error when $t_{0}=2 \pi$.

To avoid these difficulties, it is more natural to consider the problem $S_{H}$ instead of the problem $S_{H, t_{0}}$. (This was really the reason we considered the time-dependent problems over a time interval, as well as at a fixed time.) Using (2.2), Lemma 3.1, and the techniques of [9, pp. 59-60], it is easy to establish

THEOREM 4.4. (i) The nth minimal radius of information for the hyperbolic problem $S_{H}$ over the interval $[0, T]$ is

$$
r\left(n, S_{H}\right)=\beta_{n+1}^{(r+2 m) /(2 m)}
$$

so that

$$
r\left(n, S_{H}\right)=\Theta\left(n^{-(r+2 m) / N}\right) \quad \text { as } n \rightarrow \infty .
$$

(ii) The information $N_{n}$ given by (4.1) is nth optimal information for the hyperbolic problem $S_{H}$. 
(iii) Let

$$
\varphi_{n}\left(N_{n} f\right)(t)=\sum_{j=1}^{n} \frac{1-\cos \left(\sqrt{\beta_{j}} t\right)}{\beta_{j}}\left(f, z_{j}\right) z_{j} \quad \forall(f, t) \in \dot{H}^{r}(\Omega) \times[0, T] .
$$

Then $\varphi_{n}$ is an nth minimal-error algorithm for the hyperbolic problem $S_{H}$.

As before, the algorithm given by this theorem may be difficult to implement. Again, it turns out that there is a finite element method that is (to within a constant) an minimal-error algorithm for the problem $S_{H}$.

4.4. Summary. We may summarize the results of this section in

THEOREM 4.5. If $S$ is any one of $S_{E}, S_{P, t_{0}}, S_{P}$, or $S_{H}$, then

$$
r(n, S)=\Theta\left(n^{-(r+2 m) / N}\right) \text { as } n \rightarrow \infty \text {, }
$$

whereas

$$
r\left(n, S_{H, t_{0}}\right)=O\left(n^{-(r+2 m) / N}\right) \quad \text { as } n \rightarrow \infty .
$$

5. Complexity of Related Problems. In this section, we show that the $\varepsilon$ complexity of the problems $S_{E}, S_{P, t_{0}}, S_{P}$, and $S_{H}$ is $\Theta\left(\varepsilon^{-N /(r+2 m)}\right)$ as $\varepsilon \rightarrow 0$, while the $\varepsilon$-complexity of the problem $S_{H, t_{0}}$ is $O\left(\varepsilon^{-N /(r+2 m)}\right)$.

We use the model of computation of [9, Chapter 5]. Informally, this means that linear functionals can be computed in finite time and that the cost of an arithmetic operation is unity. For $\varepsilon>0$, the $\varepsilon$-complexity of a problem $S$ is then defined to be

$$
\operatorname{comp}(\varepsilon, S)=\inf \{\operatorname{cost}(\varphi, N): e(\varphi, N, S)<\varepsilon\},
$$

where $\operatorname{cost}(\varphi, N)$ is the cost (under this model of computation) of the algorithm $\varphi$ using information $N$.

Remark 5.1. Note the difference between $\operatorname{cost}(\varphi, N)$ and $\operatorname{comp}(\varepsilon, S)$. The former is the cost of a specific algorithm, whereas the latter is the minimal cost among all algorithms that solve the problem to within $\varepsilon$. This means that problem complexity is independent of any particular algorithm, and is thus an invariant of the problem.

Using the results of Section 4 along with those of [9, Chapter 5], we find

THEOREM 5.1. If $S$ is one of $S_{E}, S_{P, t_{0}}, S_{P}$, or $S_{H}$, then

$$
\operatorname{comp}(\varepsilon, S)=\Theta\left(\left(\frac{1}{\varepsilon}\right)^{N /(r+2 m)}\right) \quad \text { as } \varepsilon \rightarrow 0,
$$

whereas

$$
\operatorname{comp}\left(\varepsilon, S_{H, t_{0}}\right)=O\left(\left(\frac{1}{\varepsilon}\right)^{N /(r+2 m)}\right) \quad \text { as } \varepsilon \rightarrow 0 .
$$

So, the problems $S_{E}, S_{P, t_{0}}, S_{P}$, and $S_{H}$ all have the same complexity, which is no better than the complexity of the problem $S_{H, t_{0}}$.

6. Summary, Extensions, and Open Questions. We have shown that it is not generally true that parabolic problems are significantly easier than elliptic problems, and that elliptic problems are significantly easier than hyperbolic problems. We have shown a stronger result, namely that certain related elliptic problems, parabolic problems, and hyperbolic problems solved over a time interval all have the same complexity, while the complexity of hyperbolic problems solved out to a particular time is not greater than that of the other problems. 
This leads one to ask whether the result on the complexity of partial differential equations noted in [9] is an isolated result, or an example of a more general situation. We feel that the latter may be true. Indeed, consider the parabolic problem

$$
\begin{aligned}
\partial_{t} u+L u=0 & \text { in } \Omega \times(0, T), \\
\partial_{\nu}^{j} u=0 & \text { on } \partial \Omega \times[0, T](0 \leq j \leq m-1), \\
u(\cdot, 0)=f & \text { in } \Omega,
\end{aligned}
$$

solved out to time $t=t_{0}$, the class of problem elements once again being the unit ball of $\dot{H}^{r}(\Omega)$. The $n$th minimal error for this new problem is

$$
\Theta\left(e^{-(n+1)^{2 m / N} t_{0}} n^{-r / N}\right) \text { as } n \rightarrow \infty
$$

and the $\varepsilon$-complexity is

$$
\Theta\left(\left(\ln \frac{1}{\varepsilon}\right)^{N / 2 m}\right) \quad \text { as } \varepsilon \rightarrow 0 .
$$

(Note that changing the way the data is used from a forcing term to initial data drastically altered the complexity. We have also observed this for hyperbolic problems.) Unfortunately, we have not succeeded in generalizing the results of [9] to related elliptic and hyperbolic problems.

Finally, note that we required that the data belong to $\dot{H}^{r}(\Omega)$ for technical reasons. However, it is more usual to assume that the data is in $H^{r}(\Omega)$ (i.e., to not require the data to satisfy any boundary conditions), especially for elliptic problems (see, e.g., [8, Theorem 8.5] and the results of [10]). Do the results of this paper still hold when the data is in $H^{r}(\Omega)$ instead of $\dot{H}^{r}(\Omega)$ ?

Acknowledgments. I would like to thank J. F. Traub (Columbia University) and G. Wasilkowski (University of Warsaw and Columbia University), as well as the referee of this paper, for their comments.

Division of Science and Mathematics

Fordham University/College at Lincoln Center

113 West 60th Street

New York, New York 10023

Department of Computer Science

Columbia University

New York, New York 10027

1. S. Agmon, Lectures on Elliptic Boundary Value Problems, Van Nostrand, Princeton, N.J., 1965.

2. I. BABUŠKA \& R. B. KELLOGG, "Nonuniform error estimates for the finite element method," SIAM J. Numer. Anal., v. 12, 1975, pp. 868-875.

3. JU. M. BEREZANSKII, Expansions in Eigenfunctions of Self-Adjoint Operators, Transl. Math. Monographs, vol. 17, Amer. Math. Soc., Providence, R.I., 1968.

4. G. FAIRWEATHER, Finite Element Galerkin Methods for Differential Equations, Lecture Notes in Pure and Appl. Math., vol. 34, Marcel Dekker, New York, 1978.

5. B. Z. KACEWICZ \& G. WASILKOWSKI, "How powerful is continuous nonlinear information for linear problems?. (In preparation.)

6. S. G. KREIN AND Y. I. Petunin, "Scales of Banach spaces," Russian Math. Surveys, v. 21,1966, pp. $85-160$. 
7. D. E. KNUTH, "Big omicron and big omega and big theta," SIGACT News, April, 1976, pp. 18-24.

8. J. T. ODEN \& J. N. REDDY, An Introduction to the Mathematical Theory of Finite Elements, Wiley-Interscience, New York, 1976.

9. J. F. TRaub \& H. Woźniakowski, A General Theory of Optimal Algorithms, Academic Press, New York, 1980.

10. A. G. Werschulz, Finite Element Methods are Not Always Optimal, Mathematics Research Report 82-11, University of Maryland, Baltimore County, June, 1982. To appear in Adv. in Appl. Math.

11. M. F. WHEELER, "A priori $L^{2}$-error estimates for Galerkin approximations to parabolic partial differential equations," SIAM J. Numer. Anal., v. 10, 1973, pp. 723-759. 